\title{
Enhancing high performance of emergency unit by improving the management emergency team system of the Plateau State Specialist Hospital Nigeria
}

\author{
Elizabeth Y.S Coker-Farrell ${ }^{* 1}$, Fabong Jemchang Yildam ${ }^{2}$, Moses Farrell Luka ${ }^{1}$, Hikmet Secim ${ }^{1}$ \\ ${ }^{1}$ Institute of Graduate Studies, Cyprus International University, Nicosia, Mersin, Turkey \\ ${ }^{2}$ Accidents and Emergencies/General Outpatient Department, Plateau State Specialist Hospital, Jos, Plateau State, Nigeria
}

Received: June 2, 2015

DOI: $10.5430 /$ jha.v4n6p123
Accepted: July 30, 2015

Online Published: October 10, 2015

URL: http://dx.doi.org/10.5430/jha.v4n6p123

\begin{abstract}
There are challenges with the management of the emergency units of healthcare organizations in Nigeria; these have been observed from the high mortality rate, high level of left before examination (LBE) of the patients, low response time to acute care patients and poor turnaround time by the healthcare givers to address emergency situations. Therefore there is an urgent need to address the situation and solve the problems best ways possible by improving the management emergency team (MET) system of the healthcare organization. These was achieved by quantitative methodology application through the distribution of questionnaires, interviews and personal observation of the situation, hence improving the quality and quantity of the man power which is the human resources with qualified and well trained medical personnel, adding also to the management emergency team are police protocol officers who can give police report immediately for unconscious accident victims on arrival for emergency treatment, eliminating need for unavailable consent form before treatment, adding intensive training programs for MET system, emergency hotlines and ambulance assistance for patients, adequate supplies of oxygen and facilities that are up-to-date for the emergency unit. These solutions have increased the stability of the emergency unit, lower mortality rate, increased the efficiency of the team, improved response time, higher survival rate, improving the staff efficiency and effectiveness, after which it is observed that the emergency unit is on high-performance level, with good survey feedback by patients and their relatives.
\end{abstract}

Key Words: Emergency, High-performance, Management, Team, Medical, Doctors, Plateau State Specialist Hospital

\section{INTRODUCTION}

USAID reported in 2009 that Nigeria carried out some surveys of 107 healthcare center, out of which $20 \%$ health care givers washed their hand before and after giving injection, $63 \%$ receive vaccination, $43 \%$ have no training or information on how to dispose waste, $32 \%$ use an off-site disposal method and $20 \%$ burn waste of theirs in the open hole which burn freely to the air, this shows that there are miles to be cov- ered in achieving a stable and good healthcare environment and making sure our emergency units are safe and secured for the patients to receive adequate treatment. ${ }^{[1]}$

In order to improve the chances of survival, there's need to improve on the performance level of the emergency for quality of life for both adult and children; the emergency unit of the hospital is responsible to provide initial urgent treatments for patients with severe form of illness and injuries,

\footnotetext{
*Correspondence: Elizabeth Y.S Coker-Farrell; Email: mattbenny2000@yahoo.com; Address: Institute of Graduate Studies, Cyprus International University, Nicosia, Mersin 10, Turkey.
} 
therefore minimizing the level of risk management contend in the healthcare organization. ${ }^{[2]}$

It has been observed in Nigeria that about 54 percent of the patients end up at the hospital emergency as a last resort after seeking help elsewhere, like from traditional healthcare givers, prayer houses, dealers in drugs and so on, due to self-acclaimed knowledge, belief, lacking of enlightenment and ignorance, or sometimes because they cannot afford the financial implications of the modern healthcare centres as they belief their other options are cheaper. A good healthcare organization should be centered on patients, very safe, affordable, timely, efficient and effective. ${ }^{[3]}$

\subsection{Challenges faced by the emergency departments of Plateau State Specialist Hospital Nigeria}

The major challenge of the emergency unit is improper implementation of the management emergency team system, in which in the case of Plateau State Specialist Hospital (PSSH), a formal management emergency team (MET) system for the emergency department (ED) is non existence, inadequate or poorly manned human resources, making it to lack complete resources with under qualified healthcare staff with improper training and development of skills. It has been observed that the emergency unit of the PSSH Nigeria do not have an emergency medicine physician, who is a professional in the field of emergency unit, someone that is readily available to give urgent attention to an acute critical situation and resuscitate the patients to a point of stability.

Secondly, just like a need to include an emergency medicine physician, a police protocol officer should be included in the MET system, in Nigeria, when an accident occurs, especially a traffic road accident, the victims are rushed to the hospital emergency unit by the eye witnesses and kind hearted people due to absence of ambulance or available hotlines to reach the hospital's ambulance, thus are not allowed to sign a consent form to secure the urgent commencement of treatment, and sometimes due to delay to reach out to the victim's relatives when patients are unconscious, a police officer is required to give a police report to guarantee the commencement of treatment, most times the patients die in the process of delay in signing off the consent form for their treatment as most of them are unconscious in the situation.

Thirdly, inadequate facilities and equipments at the ED, most of the equipments are either outdated or faulty and most times absence of the needed equipments. Presence of oxygen at all times cannot be over emphasize, but our case studied hospital here do not have these equipments standby and has become a major challenge which have caused lost of lives.

Lastly, the level of power supply is a challenge and a big 124 issue in Nigeria, the absence of adequate and uninterrupted power supply to the hospitals has been challenging, some patients have lost their lives due to this problem, either in the middle of medical attention, the current goes off or there is no current at all to start a treatment, especially those that need presence of electric current before treatment commencement, like surgeries.

From the above listed challenges, there is need to urgently address the problems associated in PSSH, as they are faced with all these challenges, and eliminating these challenges means improving to a high performing emergency unit for the hospital, increasing the survival rate and decreasing the mortality rate for patients brought to the emergency. The essence of this research to help improve health care performances at the hospital emergency unit, in terms of quality healthcare service rendering, adequate and proper emergency equipment, with adequate and well trained healthcare givers (man power), protocol observations, security presence and general presence of management emergency team leadership and it's necessity, which summarizes the improvement for the MET systems of the hospitals.

\subsection{Importance of the research}

To have the enlightenment and knowledge of the situation currently at the healthcare emergency unit of PSSH, to efficiently know those qualified to be healthcare personnel and their specializations, to understand what and who makes up for the management emergency team and how to improve on the system or formally bring it into existence in this case where it is not existing, to identify the modes of practices and how the patients are attended to at the PSSH emergency unit and to add knowledge to the operations of the emergency unit of the hospital.

\subsection{Research hypotheses}

The patients are the most vulnerable in most situation of emergency, therefore it is very important to access the knowledge, practice, behaviors and attitudes of the healthcare givers towards making the emergency high performing, for effectiveness and efficiency of the outcome. Formulating the hypothesis includes, data collection and analysis through personal observation of the unit, interviews, numerical data analysis of the questionnaires, data testing of the simulation model of the arena to validate and verify results, carrying out experiments in the arena to identify places to investigate, evaluate and input parameter changes. Longo, ${ }^{[4]}$ said to ensure that patient safety is taken into consideration, all important steps to ensure there is compliance, improvement and adherence to procedures.

H1: Management leadership directly affects the MET system

ISSN 1927-6990 E-ISSN 1927-7008 
of the emergency unit of PSSH;

H2: Improvement of facilities and equipments of the ED directly affects the MET system, leading to efficiency performance of the ED of PSSH;

H3: Increased training for the ED staffs positively affects the MET system;

H4: Skills scale positively affects the MET system, improving on the performance of the ED of PSSH.

Hypothesis and correspondence to research design models were as follows (see Figure 1):

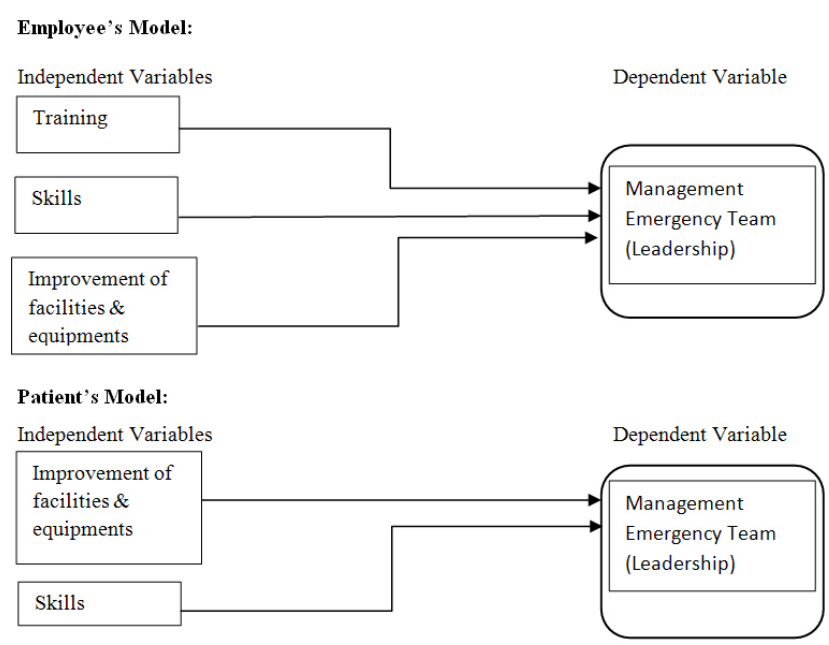

Figure 1. Research design models

\section{METHOD}

The quantitative research approach is implemented, through personal observations, interviews of healthcare personel, questionnaires distribution to collect direct feedback from healthcare givers and the patients, to view method for analyzing data from research design, population study, data collection procedure and instruments for data collection.

\subsection{Research design}

Every research is centered on data, solid accurate data for that matter, the target here are response from all employees of the ED of PSSH, and some data from patients too, with variable options weight as Strongly Disagree (S.D: 1); Disagree (D: 2); Neutral (N: 3); Agree (A: 4); Strongly Agree (S.A: 5) used in the questionaires. Other datas were collected through personal observations, interviews, field test, statistical report, written reports, oral reports, computer assisted interviews and questionnaires. The most important thing is for the collection instrument to be reliable, accurate and valid so as to meet up with the measures and values of the research design. In this case, the questionnaires were Published by Sciedu Press divided in three sections, general demographic, patients and healthcare personel sections.

\subsection{Research participants and sampling procedures}

Concentrating on the ED to get feedbacks from the medical healthcare givers, which are the doctors, nurses, sub-staff, attendants and healthcare beneficiaries who are the patients and the relatives present to help the patients with personal and emotional support. Other samples are from interviews and personal observations.

\subsection{Measures and interventions}

Griffith and White ${ }^{[5,6]}$ analyzed some necessary procedural steps needed to successfully outline the emergency diagnosis flow steps, these steps listed below will give us an idea on the summary for patients needing attention at the ED:

Step 1: Patients or relative reach out through the emergency hotlines reporting an emergency.

Step 2: Immediate guides to resituate the patient while help is being sent.

Step 3: An emergency ambulance is sent immediately to give help and bring the patient to the ED and also giving some first aid assistance to the victims while in the ambulance.

Step 4: Patient arrives at the emergency unit, either through the ambulance assistance or by arriving themselves to the hospital.

Step 5: Life support if needed immediately.

Step 6: Review on medical history and record, complaints, observation and physical examination.

Step 7: From most to least, possible diseases are listed for different diagnosis.

Step 8: To confirm the correctness of diagnosis through the laboratory, consulting specialist, $x$-ray radiology procedures, imaging, and study.

Step 9: Treatment begins based on the correct and confirm diagnosis with the help of the results by a professional skilled healthcare giver or a referred specialist.

Step 10: Progress of treatment monitored by physical observation, listening to the patient's feedback and running tests.

Step 11: Now ask to know if the diagnosis is being effective and progressive, if yes, discharge the patient unless there is further need for medical attention in or within the hospital, if no progressive diagnosis response, kindly return to step 6 and re-run the loop.

Step 12: Bill settlements. ${ }^{[7,8]}$ 
To summarize the above steps is in an algorithm, Figure 2 shows the algorithm of the emergency intervention on example of a patient with an acute chest pain-attack. Patients arrive the emergency either by waking in, though ambulance or brought in by a third party, at arrival the triage nurse examine each case and place them on a priority rank, ED is not a department of first come, first serve, it is a department that the worst critical cases are attended to first and the least critical cases come last, by doing so with the help of the triage nurse, the system becomes effective and more efficient therefore enhancing the performance of the system to attain the goals to achieve. ${ }^{[5,6]}$

\section{RESUlts}

The results have been progressive and a great improvement have been observed after testing the datas collected and taking measures of implementing the hypothesis, as from analyzing the data, corrections were made to the way the hospital handle patient flow, giving a better and clearer picture of the ED, it's clear to know and identity areas that require improvement, these scales were tested and derived from our results which we analyzed with the SPSS software. Leadership management, training, skills and improvement of facilities scales, starting with the hospital making a better schedule and arranged shifts that matches the arrival of the patients, so that there will be an effective distribution of emergency attention, improving the structural settings so as to maximize the patients output results, the level of the response and performance has improved and it has been observed that:

- Increased survival rate by $30 \%$ from initial state.

- Low rate of left before examination (LBE) patients, which have also increased the revenue level of the hospital.

- High response time from the health caregivers, being readily available and eliminating time wasting.

- Elimination of un-attendance to unconscious patients due to presence of the police protocol officer.

- High spirited and ethical MET members, rendering their services at their best.

- Improve up-to-date equipment and facilities to handle advance life support.

- Scheduled often training to always improve on the knowledge of the MET.

- Increased budget and funding to the emergency unit of the hospital.

- Availability of stand by electricity to eliminate any delay to carry out surgeries urgently.

- Improvement in emergency unit extension support, of the ambulance and first aid.
- More staffing at the emergency hotlines numbers, with shifts and availability of a personnel at all time.

- Supply and maintenance of support facilities like the fax machine, portable x-ray, advance life support and results interpreters.

- Addition of qualified skilled professionals to the MET system have increased patient throughput by $40 \%$.

Table 1 and Table 2 shows the data analyzed from the employee's data collected and patient's data collected, the correlations between the scales correlate significantly.

Table 1 shows the correlation analysis of the employee research data for the dependent scale management and all its independent scales training, improvement of facilities and skills. From the analysis the scale improvement correlate highly with management scale with a value of .531 and very significant at 1 percent, also skills scale correlate with the management scale with a value of .441 with probability of significant at .007 , also the training scale correlate with the skills scale with a value .486 with a significant probability value of .003 , therefore we can conclude that our measuring scales are valid, improvement on each of them will increase the efficiency of performance of the ED of PSSH, hence they should be implemented, as there correlation values significantly correlates.

The results from this research and study search and appraise the level of management involved in the MET system of PSSH, Plateau state, Nigeria, in order to increase the efficiency level of performance of the ED. Due to the constrain of the case studied area of the hospital which is the ED only, two categories of sample size were considered, and data was extracted from both categories which are the employees and patients. The employee sample size of the ED is about 40 staffs while having access to one patient per day for the period of the research due to the critical conditions of the patients and inability to respond immediately to the questionnaires, there are approximately 30 numbers of patients involved in the patient's sample.

For the employees, random samples were collected from all categories of staffs at the ED, which involved the doctors, nurses, laboratory technicians and sub-staffs.

For the completed questionnaires, the SPSS software was used to analyzed the collected data and both returned with a cronbach's alpha value above 0.6 which is the least required value for a reliability test, the values for the test are 0.764 and 0.609 for the patient's data and the employee's data respectively, giving us a relief level of trust that we can rely on the data collected and analyzed. 


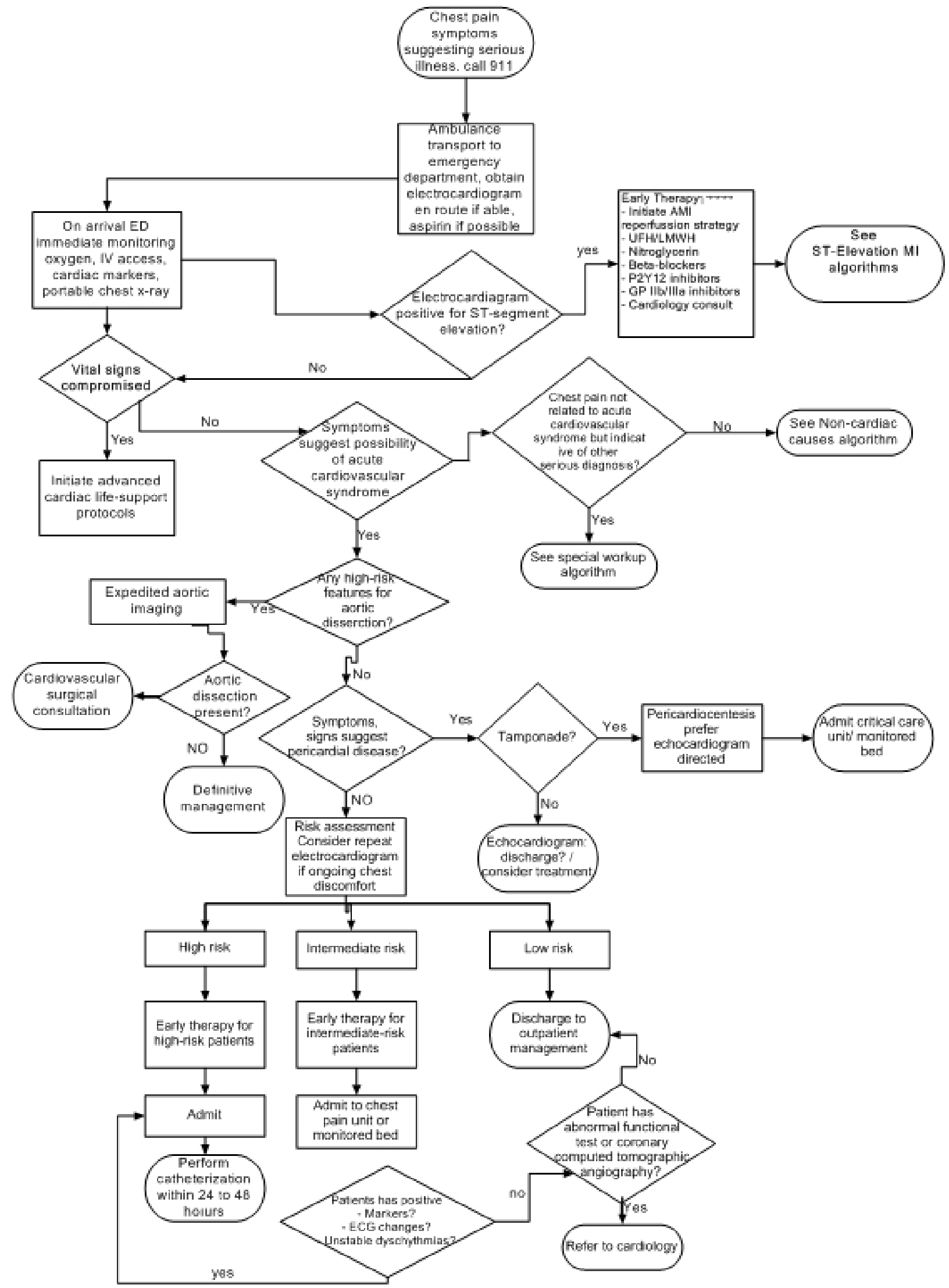

Figure 2. Emergency intervention algorithm 
Table 1. Correlation of management versus all scales for employee's data

\begin{tabular}{|c|c|c|c|c|}
\hline & \multicolumn{4}{|c|}{ Pearson Correlation ( $p$-value) } \\
\hline & Management & Improvement & Training & Skills \\
\hline Management & 1 & $0.531^{* * *}(.001)$ & $0.232(.173)$ & $0.441^{* *}(.007)$ \\
\hline Improvement & $0.531^{* *}(.001)$ & 1 & $0.112(.517)$ & $0.037(.832)$ \\
\hline Training & $0.232(.173)$ & $0.112(.517)$ & 1 & $0.486^{* *}(.003)$ \\
\hline Skills & $0.441^{* * *}(.007)$ & $0.037(.832)$ & $0.486^{* * *}(.003)$ & 1 \\
\hline
\end{tabular}

${ }^{* *}$ Correlation is significant at the .01 level (2-tailed); $\mathrm{N}=36$

\subsection{Recommended implemented results for PSSH}

- MET system should immediately be set up for the ED of PSSH, in order to reinforce the management's need to motivate and update the employees as they play very important role the health sector. ${ }^{[9]}$

- The hospital management should increase the staffing capacity of the ED as human resources are the most important resources of every people oriented organization.

- Standard organized procedures should be implemented and duly followed in caring for emergency patients. ${ }^{[10]}$

- The use of shortcuts to care for emergency patients must be done only by highly skillful and qualified trained healthcare personnel at only recommended times.

- Periodic and adequate training and knowledge must be made available to all healthcare personnel, as it is very important they are being updated on the latest in knowledge and new diseases discovered. ${ }^{[11]}$

- The level of team cohesion among staff should be high, as it is important the staff bond closely or to a comfortable level so that they can conveniently walk together for a productive high performance, team building can be apply here.

- The bridge between the hospital management and the ED management should be narrowed to the minimum, for free and easy communication and coordination. ${ }^{[12]}$

- There should be implemented policy to guide staff's working environment, hence equity must be applied to all staff especially when a policy is bridged and careless mistakes occurred.

- ED staffs must all be carried along and notified first on all changes concerning the department before other units of the hospital are informed, this is a sign of respect for the ED staffs.

- Unstructured problems as they occur must be changed with structured solutions to prevent re-occurrence and inability of countenance. ${ }^{[13]}$

- The hospital and ED management must motivate the ED staffs adequately in terms of rewards and wages, to minimize staff turnover, job dissatisfaction and ab- senteeism. $^{[14]}$

- The staff's ideas and suggestion towards positively improving the performance of the ED without any biased motives should be highly considered and recommended.

- The ED department must be equip with needed necessary facilities and equipments of advance life support machines that are up to date with required training for those to operate it. ${ }^{[15]}$

- The ED staff must have the skills and training to behave professionally and being very supportive and responsive to the patients and their needs.

- Hygiene of the ED must be improved and necessary renovations must be carried out to restore adequacy.

- Special and professionals in some distinct areas must be employed to handle the area of pain management, emergency medicine doctor, triage doctor and nurse, protocol officer etc.

- Inspection, supervision and regulations of the ED must be done periodically to make sure the ED adhere to the laws and professional regulations that binds the healthcare practices at the emergency. ${ }^{[16]}$

- Hotlines should be designed for the ED of PSSH, so that patients can reach and call for help at emergency situations.

- The ED should be equipped with ambulance and drivers for the ambulance should be employed to serve.

- Addition of a PSO (Patient's Safety Officer) to ensure compliance and adhering to guidelines of practices. ${ }^{[17]}$

Table 2 reveals data analyzed from the patients, and from their point of view, the skills scale correlate with the management scale at 0.640 with significant rate of .000 , also the improvement scale correlate with the management scale at 0.640 with significant value of .000 , the correlations are emphasized with the stared value showing they are highly significant too, these scales should be increase to improve the efficiency and performance of the ED of PSSH.

Table 2. Correlation of management versus all scales for patient's data

\begin{tabular}{lllll}
\hline \multicolumn{5}{l}{ Pearson Correlation (p-value) } \\
\hline & Management & Skills & Improvement & Training \\
Management & 1 & $0.640^{* * *}(.000)$ & $0.640^{* * *}(.000)$ & $-0.106(.584)$ \\
Skills & $0.640^{* * *}(.000)$ & 1 & $1.000^{* * *}(.000)$ & $-0.333(.078)$ \\
Improvement & $0.640^{* * *}(.000)$ & $1.000^{* * *}(.000)$ & 1 & $-0.333(.078)$ \\
Training & $-0.106(.173)$ & $-0.333(.078)$ & $-0.333(.078)$ & 1 \\
\hline${ }^{* * *}$ Correlation is significant at the .01 level (2-tailed); $\mathrm{N}=29$ &
\end{tabular}

\section{Discussion}

The primary hypothesis is the management leadership which can be distinguished from the secondary hypothesis of train- 
ing, skills, improvement of facilities and equipments.

Based on the results found in the research and using our hypothesis, we considered four scales to analyze our results with:

(1) Management Emergency Team (Leadersip) Scale: This scale focus solely on management issues of the $\mathrm{ED}$, it is said to be the dependent scale in which the other scales are independent to, when analyzed, the cronbach's alpha value for the employee's data is 0.609 and for the patient's data is 0.764 , the KMO value is 0.664 and 0.500 for employee and patient's data respectively, while the significant level is .000 and .000 for both categories, this shows that the management scale is very significant and a necessary scale to measure the performance of the MET system and PSSH ED. The management scale also analyze the staffing issues of the department and the management deficiency, therefore it's concluded that management affects the performance of the ED of PSSH directly and the situation of PSSH ED is very critical, as they do not have a formal MET system for the ED and so the informal management which consist of the head doctor of the department, head nurse and some other doctors and nurse come together informally to share ideas and organize a routine to run the department, unfortunately when need arises and they relate it to the hospital management, they hardly get response or feedback most times, and at those often times they do get a feedback, it is usually after a very long time, because they are not a formal structure on the hospital hierarchy structure. Therefore we can say the first and major challenge of the ED of PSSH is lack of a formal management to control the situation of the system, hence leading to poor performances of the ED.

(2) Improvement of Facilities and Equipments Scale: This scale is independent to the management dependent scale, this scale helped to analyze the level of availability of facilities at the ED of PSSH and was found out that the hospital ED have little or no emergency facilities, and for the department to function adequately, this situation must be addressed. When this scale was analyzed on the SPSS software, the KMO value returned as 0.555 and the significant value is .006 for the employee's data while for the patient's data, the KMO value is 0.606 and the significant value is .002 , which means the scale is very significant and correlate with the dependent scale when the component matrix was carried out, as the determinate value of correlation is 0.685 for employee's data and 0.559 for the patient's data.
(3) Training Scale: For this scale its' considered data extracted from the employees only, because the patients do not have the expertise to really judge the training ability of the employees because of the profession of the practice. Therefore from the data extracted from the employees the KMO value is 0.500 and the significant value is .000 which clearly shows its significant to the study and when the correlation matrix was carried out, it correlate and returned a determinate value of 0.420 against a recommended value of 0.00001 . The training of the employees should be more often and updated as the health environment is changing frequently and vastly. The lack of regular and needed training is affecting the performance of the ED of PSSH obviously and increase the efficiency and performance of the ED, then the training scale can be expressed more.

(4) Skills Scale: This scale as the immediate above will be considered on the employee data only for the same reason, hence the extraction from the factor analysis shows a KMO value of 0.560 and significant level of .25 , which means it is significant but then not very significant because the value is not so close to .000 and so we found out the skills scale varies directly with the ED performance but inversely with the ED MET system of the ED of PSSH. The determinate value at correlation matrix is 0.754 .

\section{Conclusion}

To experience a high performance at the emergency unit of our healthcare organizations then we must implement all measures as found in the case of this research, it was observed that from this research that most research look at improving the system already on ground and in use, ${ }^{[17,18]}$ which is very important but this research further stress the need to expand the MET system by adding some keys roles that do not exist in the emergency team especially addition of a police protocol officer to the team. ${ }^{[19,20]}$ To also emphasize on some important issues solved is the addition of an emergency physician whose main job is to attend to emergency situations in the hospital instead of depending on a general doctor in the hospital who could be busy at other departments of the hospital in some emergency cases, to also include roles is a triage nurse, to systematically avail treatment procedures to available healthcare personnel and equipment on bases of most needed, that is the most critical conditions first and not a first come first serve situation because some conditions are more critical than others, and so by carefully accessing the patients and analyzing all their complains, then they can be set to most critical categories by triage priority, then also to add PSO, that is a patient safety officer, who manages, lead, 
control and plan the procedures of the ED by ensuring the maximum level of value addition, safety measures and compliance to risk management. ${ }^{[21,22]}$ These are to summarize urgent needs at the PSSH ED in Nigeria.

\section{ACKNOWLEDGEMENTS}

We acknowledge God almighty for the capacity He has given us to carry out this research, the team acknowledge the support of the PSSH in data collection process and support of well-respected people who have contributed immensely to the success of this study. Thank you for your patience and generous support.

\section{REFERENCES}

[1] Musa I. Appraisal of knowledge, attitude and practice of biomedical waste management among healthcare workers in Murtala Muhammad specialist hospital Kano state, Nigeria [Masters of Art]. Cyprus International University; 2014. 2-3.

[2] Carroll R (ed.). Risk Management Handbook for Health Care Organizations, Student Edition, $5^{\text {th }}$ Edition. San Francisco: Jossey-Bass Center for Health Care Strategies, Inc. Online information; 2009 [accessed November 2014]. Available from: www . chcs .org/usr_d oc/P4P_Resource_Paper.pdf

[3] Black J, Miller D. The Toyota Way to Healthcare Excellence: Increase Efficiency and Improve Quality with Lean, Chicago: Health Administration Press; 2008.

[4] Longo DR, Hewett JE, Ge B, et al. The Long Road to Patient Safety: A Status Report on Patient Safety Systems. JAMA. 2005; 294(22): 2858-65. PMid: 16352793. http://dx.doi.org/10.1001/jama. 294.22 .2858

[5] Griffith JR, White KR. The Revolution in Hospital Management. Journal of Healthcare Management. 2012; 50(3): 170-190.

[6] Griffith JR, White KR. The well-managed healthcare organizationChicago Illinois: AUPHA hap; 1999. 13-22.

[7] Spath PL (ed.). Beyond Clinical Paths: Advanced Tools for Outcomes Management. Chicago: American Hospital Publishing; 1997. 5-8.

[8] Spath PL. Leading Your Healthcare Organization to Excellence: A Guide to Using the Baldrige Criteria. Chicago: Health Administration Press; 2005.

[9] Christianson JB, Warrick LH, Netting FE, et al. Hospital CaseManagement: Bridging Acute and Long-Term Care. Health Affairs. 1991; 10(2): 173-184. PMid: 1909290. http://dx.doi.org/10.1377 /hlthaff.10.2.173

[10] Groszkruger JDD. Perspectives on Healthcare Reform: A year later, what more do we know. American Society for Healthcare Risk Management. 2010 [Accessed 16 December, 2014]. Available from: http://www.ashrm.org/ashrm/education/developm ent/journal/Journal\%20Article_HealthcareReform.pdf

[11] Hasselman D. Provider Incentive Programs: An Opportunity for Medicaid to Improve Quality at the Point of Care. Institute of Medicine,
Committee on Quality of Health Care in America (2000). To Err is Human: Building a Safer Health System, edited by Kohn LT, Corrigan JM, Donaldson MS. Washington, DC: National Academies Press; 2009. 29-38.

[12] Kelly DL. Applying Quality Management in Healthcare: A Systems Approach, $2^{\text {nd }}$ ed. Chicago: Health Administration Press; 2006. 136-143.

[13] Lambert MJ. Leading a Patient-safe Organization. Chicago: Health Administration Press; 2004. 243-251. PMid: 15574113.

[14] Proenca EJ. Team Dynamics and Team Empowerment in Health Care Organizations. Health Care Management. Review. 2007; 32(4): 370-78. PMid: 18075446. http://dx.doi.org/10.1097/01.HM R.0000296786.29718.86

[15] Schuster MA, McGlynn EA, Pham CR, et al. The Quality of Health Care in the UnitedStates: A Review of Articles Since (1987). Appendix A of Crossing the Quality Chasm: A New Health System for the $21^{\text {st }}$ Century. Washington, DC: National Academies Press; 2001

[16] Spear SJ. Fixing Health Care from the Inside, Today. Harvard Business Review. 2005; 83(9): 78-91. PMid: 16171213.

[17] Stein K. Emergency medicine: Emergency department protocols: practical pain management. 2001

[18] Stephen HT (ed.). Management of pain in the emergency department: ISRN emergency medicine; 2013.

[19] Time Series Analysis of Emergency Unit. The Guardian Nigeria Information. 2005.

[20] White RK, Griffith RJ (ed.). The Well-Managed Healthcare Organization: Health Administration Press; 2012. 141-167.

[21] Williams FG, Warrick LH, Christianson JB, et al. Critical Factors for Successful Hospital-BasedCase Management. Health Care Management Review. 1993; 18(1): 63-70. http://dx.doi.org/10.1097 /00004010-199324000-00007

[22] Wardhani V1, Utarini A, van Dijk JP, et al. Determinants of Quality Management Systems Implementation in Hospitals. Health Policy. 2009; 89(3): 239-51. PMid: 18752866. http://dx.doi.org/10. $1016 / j$.healthpol.2008.06.008 